\title{
Pomegranate protects renal proximal tubules during gentamicin induced-nephrotoxicity in rats
}

\author{
Hayder M. Al-kuraishy, Ali I. Al-Gareeb, and Marwa S. Al-Naimi
}

Department of Clinical Pharmacology, Medicine and Therapeutic, Medical Faculty, College of Medicine, Al-Mustansiriya University, Baghdad, Iraq.
${ }^{*}$ Correspondence to Hayder M. Alkuraishy (email: hayderm36@yahoo.com)
(Submitted: 14 October 2018 - Revised version received: 19 November 2018 - Accepted: 10 December 2018 - Published online: 26 February 2019)

Objective To evaluate the nephroprotective effect of pomegranate in gentamicin induced-nephrotoxicity in rats.
Methods Thirty Sprague-Dawley male rat were used, which randomly divided into three groups, 10 rats in each group. Group 1 ( $n=10)$ :
rats treated with distilled water for 12 days. Group 2 ( $n=10)$ : rats treated with distilled water + gentamicin. Group 3 ( $n=10)$ : rats treated
with pomegranate + gentamicin. Blood urea, serum creatinine, serum malondialdehyde (MDA), superoxide dismutase (SOD), glutathione
reductase (GSH), neutrophil gelatinase associated lipocalin (NGAL), kidney injury molecules (KIM-1), and cystatin C were measured.
Results Blood urea and serum creatinine were significantly elevated in gentamicin group compared with the control group $(p=0.007$ and
0.04) respectively. Also, estimated GFR was significantly decreased in gentamicin group compared with the control group ( $p=0.04)$.
Cystatin-C and MDA serum level were increased but SOD and GSH were decreased insignificantly in gentamicin group compared with the
control group ( $p>0.05)$. Pomegranate reduced blood urea significantly ( $p=0.002)$. Pomegranate improves endogenous anti-oxidant
capacity, since it increase GSH significantly compared with gentamicin group ( $p=0.02)$. There were insignificant effects on MDA and SOD.
Moreover, pomegranate reduce serum levels of KIM-1and NGAL in gentamicin group $(p<0.05)$.
Conclusion Pomegranate attenuates gentamicin induced-nephrotoxicity through potentiation of endogenous anti-oxidant capacity and
inhibition of inflammatory pathway.
Keywords nephrotoxicity, pomegranate, gentamicin, anti-oxidant capacity

\section{Introduction}

Nephrotoxicity is a renal-specific condition in which the excretions of toxic metabolites are accumulated due to toxic agents and drugs. In relation $20 \%$ of nephrotoxicity is induced and caused by drugs; this percentage is augmented in the elderly due to rise in the life span and poly-medications.

There are diverse mechanisms lead to nephrotoxicity, including renal tubular toxicity, inflammation, glomerular damage, crystal nephropathy, and thrombotic microangiopathy. ${ }^{2}$

Proximal renal tubular cells are in prolonging contact with drugs due to tubular reabsorption and concentration processes. Toxic agents and drugs cause potential damage to the tubular transport system through induction of free radical formation and oxidative stress which lead to tubular cell mitochondrial damage. ${ }^{3}$ The drugs that cause tubular damage are aminoglycoside, amphotercin B, antiviral like adefovir, foscarnet and cytotoxic agents like cisplatin. ${ }^{4}$ Gentamicin is an antibiotic belongs to aminoglycoside group, used for treatment of different bacterial infections, it is bactericidal acts as protein synthesis inhibitor through binding to the 30 s subunit of bacterial ribosome. ${ }^{5}$

Actually, $90 \%$ of administrated gentamicin is not metabolized by the liver and it is mainly excreted unchanged in the proximal renal tubules leading to extensive necrosis at a higher dose. ${ }^{6}$ Gentamicin enters the cell by specific ion channels and by endocytosis. Most of the cells clear gentamicin deposition by efflux it but remains concentrated at renal cortical cells. ${ }^{7}$ Overproduction of reactive oxygen species and free radicals are the main mechanism beyond the gentamicin induced nephrotoxicity via over-expression of cation transporter proteins (megalin and cubilin) at proximal renal tubules that augment the accumulation of gentamicin and free radical generations. ${ }^{8,9}$

Pomegranate (Punica granatum L.) is a potent nutraceutical herb used in prevention and treatment of different diseases.
It is wealthy in phytochemicals which are related to steroid hormones in their structures that bind estrogenic receptors which called phyto-estrogens. ${ }^{10,11}$ These compounds are activated by gastro-intestinal enzyme which act as a partial agonist on the estrogenic receptors. ${ }^{12}$ Pomegranate peel contains $50 \%$ of bioactive constituents including flavonoids, phenolics, proanthocyanide, and ellagitannins with different minerals. Pomegranate's edible part contains $10 \%$ seeds and $40 \%$ arils, arils mainly enclose anthocyanins while; the seeds contain mainly anthocyanin and glucosides. Punicic acid is the chief component of pomegranate oil. ${ }^{13}$ Pomegranate inhibits lipid peroxidation and scavenge oxidative free radicals via augmentation of tissue catalase, peroxidase and superoxide dismutase in renal and other organs. ${ }^{14}$ Keenly, pomegranate inhibits gene expression of pro-inflammatory cytokines via suppression of NF- $\kappa \mathrm{B}$ activation. Ellagic acid leads to significant inhibition of prostaglandin E2 through inhibition of COX-2. ${ }^{15,16}$

Regarding the effect of pomegranate on the kidney function, it chiefly affects renal glomeruli through induction of endothelial NO by flavonoids which enhance glomerular blood flow. In addition, pomegranate showed less effect on renal medulla with mild increase in the interstitial volume. ${ }^{17}$

Therefore, the aim of this study was to evaluate the nephroprotective effect of pomegranate in gentamicin induced-nephrotoxicity in rats.

\section{Materials and Methods}

Thirty Sprague-Dawley male rat were used, these animals were gained from the National Center for Drug Control and Research. Rats age ranged from 3 to 4 months and their body weight ranged from 200 to $400 \mathrm{~g}$. The animals were isolated as three rats in each sterilized cage and placed with suitable 
temperature $\left(22-25^{\circ} \mathrm{C}\right)$ with artificial $12 / 12$ light cycle. They were left for 1 week for adaptation without any intervention with free access to normal chow pellets and water. Human care for animals was according to the guide to the care and use of laboratory animal. After acclimatization period, weights of rats were taken and rat with wound infection were excluded then rats were randomly divided into three groups, 10 rats in each group. The study protocol and method for induction of AKI was according to Singh et al.'s ${ }^{18}$ method.

Control group $(\boldsymbol{n}=\mathbf{1 0})$ : Rats treated with distilled water (5 mL/kg, p.o.) for 12 days and on days $6-12$ received an intraperitoneal (i.p.) injection of normal saline $(5 \mathrm{~mL} / \mathrm{kg})$ daily.

Gentamicin group $(\boldsymbol{n}=\mathbf{1 0})$ : Rats treated with distilled water $(5 \mathrm{~mL} / \mathrm{kg}$, p.o.) for 12 days and on days $6-12$ received gentamicin $100 \mathrm{mg} / \mathrm{kg}$, i.p.

Pomegranate Group $(\boldsymbol{n}=\mathbf{1 0})$ : Rats treated with pomegranate $(50 \mathrm{mg} / \mathrm{kg}$, p.o.) for 12 days and on days $6-12$ received gentamicin $100 \mathrm{mg} / \mathrm{kg}$, i.p. at an interval of $1 \mathrm{~h}$.

\section{Anthropometric Measurements}

Length was measured by using graduated tape from nose to the anus (naso-anal length in $\mathrm{cm}$ ). Rat body weight was measured by using specific digital balance in grams. Body mass index equal body weight in grams over the square of length in $\mathrm{cm}$, BMI $=$ BW $(\mathrm{g}) /$ length $(\mathrm{cm})^{2}$. Estimated glomerular filtration rate (eGFR) was measured according to Schwartz formula, $\mathrm{eGFR}=k \times$ height $(\mathrm{cm}) /$ serum creatinine $(\mathrm{mg} / \mathrm{dL})$, $k=0.55 .{ }^{19}$

\section{Sample Collection}

On $13^{\text {th }}$ day, chloroform was used to anesthetize the rats and sharp scissors were used to exudate the rats. The blood sample was allowed to drain in sterile gel tube, and centrifuged for $10 \mathrm{~min}$ at $5000 \mathrm{rpm}$, so the formed supernatant layer was isolated as serum sample and kept in freezer at -20 to be assessed later.

\section{Assessment of Biochemical Variables}

Blood urea and serum creatinine were estimated by using an auto-analyzer (ILab-300-Biomerieux Diagnostic, Milano, Italy) expressed as $\mathrm{mg} / \mathrm{dL}$. Serum malondialdehyde (MDA), superoxide dismutase (SOD), glutathione reductase (GSH), neutrophil gelatinase associated lipocalin (NGAL), kidney injury molecules (KIM-1) and cystatin C were measured by ELISA kit methods according to the instruction of the kit manufacture (MyBioSource, USA).

\section{Statistical Analysis}

Statistical package for the Social Sciences Software (SPSS Inc., Chicago, IL, USA) was used for data analysis. Data of this study was presented as mean $\pm \mathrm{SD}$ and the variables were tested by using unpaired student $t$-test between control and treated groups. One-way ANOVA test with post-hoc test was used to investigate the significance of differences among different groups. The levels of significance was regarded when $p<0.05$.

\section{Results}

\section{Effects of Gentamicin on the Renal Biomarkers}

Comparison of different variables between controls and gentamicin group showed that there was statistically insignificant difference in the weight and height, of the two groups, while
BMI was significantly high in gentamicin group compared with the control group $(p=0.001)$. Both blood urea and serum creatinine were significantly elevated in gentamicin group compared with the control group ( $p=0.007$ and 0.04 ), respectively. Also, estimated GFR was significantly decreased to 11.19 $\pm 5.16 \mathrm{~mL} / \mathrm{min} / 1.73$ in gentamicin group compared with the control group $(16.89 \pm 4.21 \mathrm{~mL} / \mathrm{min} / 1.73, p=0.04)$. Regarding the oxidative stress and anti-oxidant biomarkers, MDA serum level was increased but SOD and GSH were decreased insignificantly in gentamicin group compared with the control group $(p>0.05)$. As well, KIM-1 and NGAL were significantly elevated in gentamicin group compared with the control group ( $p=0.02$ for NGAL and $p<0.01$ for KIM-1) (Table 1$)$.

Indeed, cystatin-C serum level was significantly increased during induction of nephrotoxicity by gentamicin from 0.024 $\pm 0.0005 \mathrm{ng} / \mathrm{mL}$ in the control group to $0.0280 \pm 0.0016 \mathrm{ng} / \mathrm{mL}$ in the experimental group ( $p=0.01$ ) (Fig. 1).

\section{Effects of Pomegranate on the Renal Biomarkers}

Concerning the effect of pomegranate on gentamicin inducenephrotoxicity it causes insignificant effect on the weight, height, and BMI of rats $(p>0.05)$. Pomegranate reduced blood

Table 1. Effect of gentamicin on the anthropometric variables, biochemical and inflammatory biomarkers in gentamicin induced-nephrotoxicity

\begin{tabular}{lccc}
\hline Variables & Control $(\boldsymbol{n}=\mathbf{1 0})$ & Gentamicin $(\boldsymbol{n}=\mathbf{1 0})$ & $\boldsymbol{p}$ \\
\hline Weight $(\mathrm{g})$ & $268.00 \pm 25.01$ & $288.37 \pm 34.02$ & 0.24 \\
Height $(\mathrm{cm})$ & $21.50 \pm 0.83$ & $21.99 \pm 0.88$ & 0.31 \\
BMI $\left(\mathrm{g} / \mathrm{cm}^{2}\right)$ & $0.57 \pm 0.02$ & $0.59 \pm 0.04$ & $0.0001^{*}$ \\
$\begin{array}{l}\text { Blood urea } \\
\text { (mg/dL) }\end{array}$ & $41.83 \pm 7.46$ & $56.87 \pm 9.33$ & $0.007^{*}$ \\
$\begin{array}{l}\text { Serum creati- } \\
\text { nine }(\mathrm{mg} / \mathrm{dL})\end{array}$ & $0.70 \pm 0.14$ & $1.08 \pm 0.40$ & $0.04^{*}$ \\
$\begin{array}{l}\text { Estimated GFR } \\
\text { (mL/min/1.37) }\end{array}$ & $16.89 \pm 4.21$ & $11.19 \pm 5.16$ & $0.04^{*}$ \\
MDA $(\mathrm{ng} / \mathrm{mL})$ & $289.85 \pm 44.18$ & $408.11 \pm 145.8$ & 0.08 \\
SOD $(\mathrm{pg} / \mathrm{mL})$ & $48.12 \pm 32.92$ & $26.39 \pm 16.86$ & 0.13 \\
GSH $(\mu \mathrm{g} / \mathrm{mL})$ & $15.94 \pm 2.39$ & $13.89 \pm 2.94$ & 0.18 \\
KIM-1 $(\mathrm{pg} / \mathrm{mL})$ & $73.78 \pm 16.29$ & $354.98 \pm 46.38$ & $0.0001^{*}$ \\
NGAL $(\mathrm{pg} / \mathrm{mL})$ & $15.78 \pm 3.07$ & $20.04 \pm 2.88$ & $0.02^{*}$ \\
\hline
\end{tabular}

${ }^{*} P<0.05$, unpaired $t$-test.

BMI: body mass index, GFR: glomerular filtration rate, MDA: malondialdehyde SOD: superoxide dismutase, GSH: glutathione reductase, KIM-1: kidney injury molecule-1, NGAL: neutrophil gelatinase associated lipocalin.

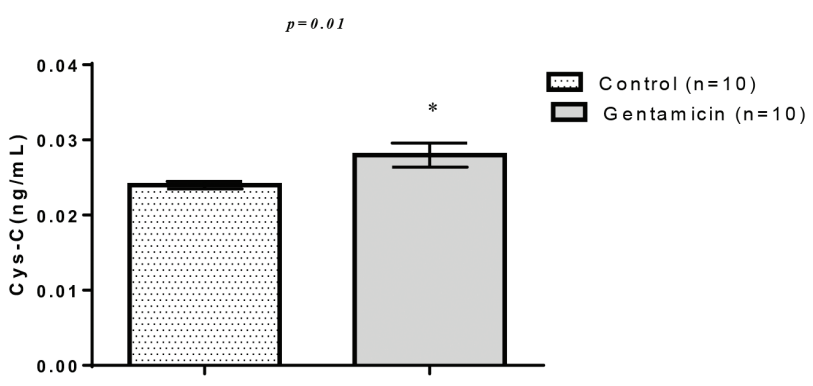

Fig. 1 Cystatin-C serum levels in gentamicin induced-nephrotoxicity $\left({ }^{*} \mathrm{p}<0.05\right)$. 
urea significantly from $56.87 \pm 9.33$ to $40.25 \pm 8.95 \mathrm{mg} / \mathrm{dL}$ $(p=0.002)$ without significant reduction of serum creatinine $(p>0.05)$ or improvement of estimated GFR. Pomegranate improves endogenous anti-oxidant capacity, since it increase GSH significantly up to $17.91 \pm 3.34 \mu \mathrm{g} / \mathrm{mL}$ compared with $13.89 \pm 2.94 \mu \mathrm{g} / \mathrm{mL}$ in gentamicin group $(p=0.02)$. There were insignificant effects on MDA and SOD. Moreover, pomegranate attenuated renal injury significantly, since it reduces serum levels of KIM-1 from $354.98 \pm 46.38 \mathrm{pg} / \mathrm{mL}$ in gentamicin group to $102.71 \pm 57.20 \mathrm{pg} / \mathrm{mL}(p=0.0001)$ (Table 2$)$.

Whereas, no significant change in cystatin- $\mathrm{C}$ in pomegranate group $(0.026 \pm 0.007 \mathrm{ng} / \mathrm{mL})$ compared with gentamicin group $0.0280 \pm 0.0016 \mathrm{ng} / \mathrm{mL}(p=0.67$ ) (Fig. 2).

Table 2. Effect of pomegranate on the anthropometric variables, biochemical and inflammatory biomarkers in gentamicin induced-nephrotoxicity

\begin{tabular}{lccl}
\hline Variables & $\begin{array}{c}\text { Gentamicin } \\
(\boldsymbol{n}=1 \mathbf{0})\end{array}$ & $\begin{array}{c}\text { Pomegranate } \\
(\boldsymbol{n}=\mathbf{1 0})\end{array}$ & $\boldsymbol{p}$ \\
\hline Weight $(\mathrm{g})$ & $288.37 \pm 34.02$ & $276.50 \pm 29.22$ & 0.46 \\
Height $(\mathrm{cm})$ & $21.99 \pm 0.88$ & $21.67 \pm 0.78$ & 0.45 \\
BMI $\left(\mathrm{g} / \mathrm{cm}^{2}\right)$ & $0.59 \pm 0.04$ & $0.59 \pm 0.45$ & 1.0 \\
Blood urea $(\mathrm{mg} / \mathrm{dL})$ & $56.87 \pm 9.33$ & $40.25 \pm 8.95$ & $0.002^{*}$ \\
Serum creatinine & $1.08 \pm 0.40$ & $0.87 \pm 0.18$ & 0.15 \\
(mg/dL) & & & \\
Estimated GFR & $11.19 \pm 5.16$ & $13.69 \pm 4.97$ & 0.34 \\
(mL/min/1.73) & & & \\
MDA (ng/mL) & $408.11 \pm 145.8$ & $371.25 \pm 66.81$ & 0.52 \\
SOD (pg/mL) & $26.39 \pm 16.86$ & $32.17 \pm 14.90$ & 0.47 \\
GSH $(\mu \mathrm{g} / \mathrm{mL})$ & $13.89 \pm 2.94$ & $17.91 \pm 3.34$ & $0.02^{* *}$ \\
KIM-1 $(\mathrm{pg} / \mathrm{mL})$ & $354.98 \pm 46.38$ & $102.71 \pm 57.20$ & $0.0001^{*}$ \\
NGAL $(\mathrm{pg} / \mathrm{mL})$ & $20.04 \pm 2.88$ & $15.29 \pm 1.54$ & $0.0011^{*}$ \\
\hline
\end{tabular}

\section{${ }^{*} p<0.01$.}

${ }^{* *} p<0.05$, unpaired $t$-test.

BMI: body mass index, GFR: glomerular filtration rate, MDA: malondialdehyde, SOD: superoxide dismutase, GSH: glutathione reductase, KIM-1: kidney injury molecule-1, NGAL: neutrophil gelatinase associated lipocalin.

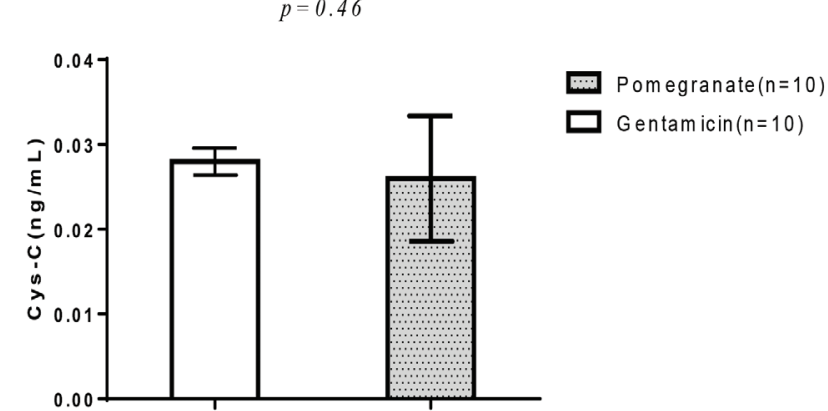

Fig. 2 Pomegranate produced insignificant reduction of cystatin-C serum levels in gentamicin induced-nephrotoxicity.

Intergroup variations illustrated significant changes in BMI, biochemical data, biomarkers of inflammatory and pro-inflammatory mediators (Table 3).

\section{Discussion}

This study illustrated that gentamicin was proficient to induce experimental nephrotoxicity in rats via significant rise of blood urea and serum creatinine with significant decline in the estimated GFR. These findings were corresponded with different recent studies. ${ }^{20,21}$

Since there were two groups of rats with minimal differences in the weight and length, control group showed low weight compared with high weight in gentamicin group which might explain high BMI in gentamicin group. Ibraheem et al.'s2 study showed that co-adminstration of gentamicin with fructose for 8 weeks encourage metabolic syndrome and increasing in the body weight and BMI which to a degree explain high body weight in gentamicin group. It has been recognized by diverse studies that the production of free radicals and induction of oxidative stress are the most imperative pathway of gentamicin induced-nephrotoxicity. So, overproduction of reactive oxygen species is connected with depletion of proximal renal tubules anti-oxidant potential which afterward developed into lipid peroxidation and tubular damages. ${ }^{23}$

Table 3. Intergroup variations in the anthropometric, biochemical and renal biomarkers in gentamicin induced-nephrotoxicity regarding the effect of pomegranate compared with the control

\begin{tabular}{|c|c|c|c|c|}
\hline Variables & Group I $(n=10)$ & Group II $(n=10)$ & Group III $(n=10)$ & ANOVA \\
\hline Weight (g) & $268.00 \pm 25.01$ & $288.37 \pm 34.02$ & $276.50 \pm 29.22$ & 0.74 \\
\hline Height (cm) & $21.50 \pm 0.83$ & $21.99 \pm 0.88$ & $21.67 \pm 0.78$ & 0.29 \\
\hline BMI $\left(\mathrm{g} / \mathrm{cm}^{2}\right)$ & $0.57 \pm 0.02$ & $0.59 \pm 0.04$ & $0.59 \pm 0.45$ & $0.001^{*}$ \\
\hline Blood urea (mg/dL) & $41.83 \pm 7.46$ & $56.87 \pm 9.33$ & $40.25 \pm 8.95$ & $0.0001^{*}$ \\
\hline Serum creatinine $(\mathrm{mg} / \mathrm{dL})$ & $0.70 \pm 0.14$ & $1.08 \pm 0.40$ & $0.87 \pm 0.18$ & $0.001^{*}$ \\
\hline $\mathrm{GFR}(\mathrm{mL} / \mathrm{min} / 1.73)$ & $16.89 \pm 4.21$ & $11.19 \pm 5.16$ & $13.69 \pm 4.97$ & $0.04^{* *}$ \\
\hline $\mathrm{MDA}(\mathrm{ng} / \mathrm{mL})$ & $289.85 \pm 44.18$ & $408.11 \pm 145.8$ & $371.25 \pm 66.81$ & $0.005^{*}$ \\
\hline $\mathrm{SOD}(\mathrm{pg} / \mathrm{mL})$ & $48.12 \pm 32.92$ & $26.39 \pm 16.86$ & $32.17 \pm 14.90$ & 0.05 \\
\hline $\mathrm{GSH}(\mu \mathrm{g} / \mathrm{mL})$ & $15.94 \pm 2.39$ & $13.89 \pm 2.94$ & $17.91 \pm 3.34$ & $0.0001^{*}$ \\
\hline KIM-1 (pg/mL) & $73.78 \pm 16.29$ & $354.98 \pm 46.38$ & $102.71 \pm 57.20$ & $0.0001^{*}$ \\
\hline Cys-c (ng/mL) & $0.024 \pm 0.0005$ & $0.028 \pm 0.0016$ & $0.026 \pm 0.0031$ & $0.0002^{*}$ \\
\hline $\mathrm{NGAL}(\mathrm{pg} / \mathrm{mL})$ & $15.78 \pm 3.07$ & $20.04 \pm 2.88$ & $15.29 \pm 1.54$ & 0.05 \\
\hline $\begin{array}{l}{ }^{*} p<0.01 . \\
{ }^{* *} p<0.05 \text {, one-way ANOVA te } \\
\text { BMl: body mass index, GFR: gl }\end{array}$ & 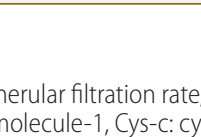 & $\mathrm{c}_{\mathrm{c}} \mathrm{s}$ & 1. & \\
\hline
\end{tabular}


Therefore, serum level of MDA is elevated while; SOD and GSH are reduced in different models of gentamicin induced-nephrotoxicity as illustrated by Hajihashemi et al. $\mathrm{s}^{24}$ study that confirmed the protective effect of hydroalcoholic extract of Zataria multiflora in reduction of MDA with significant effect in rise of anti-oxidant enzyme activities. Despite these findings, gentamicin in this study elevates MDA serum levels, reduced SOD and GSH but not significantly which might due to insufficient gentamicin dose, small sample size or short duration of the experimental study.

This study also illustrated significant effect of gentamicin in rise KIM-1 and NGAL sera levels as correspond with Luo et al.s study that showed both KIM-1 and NGAL sera level are sensitive and specific biomarkers and correlated with renal histopathological changes during gentamicin induced-nephrotoxicity within 7 days. The increment in those biomarkers is time and dose dependent due to progressive gene expression of KIM- 1 and NGAL. ${ }^{25}$

Usually, renal tissues expresse a low level of KIM-1 but subsequent to the renal injury, KIM-1 gene expression is noticeably up-regulated at renal proximal convoluted tubules. Moreover, KIM-1 serum levels are more sensitive than NGAL for development of acute renal injury. ${ }^{26}$

Neutrophil gelatinase associated lipocalin is a protein expressed at renal proximal convoluted tubules which also up-regulated after renal ischemia and injury. It detects in vitro and in vivo tubular damage, since it rise within $2 \mathrm{~h}$ of renal ischemia thus it regarded as a reliable biomarker for detection early acute renal injury as it correlated with the severity of renal damage. Indeed, urinary NGAL can differentiate between intrinsic and prerenal causes of acute renal injury as urinary NGAL $>104 \mu \mathrm{g} / \mathrm{L}$ indicate intrinsic cause while level $<47 \mu \mathrm{g} / \mathrm{L}$ indicate a prerenal cause of acute renal injury. ${ }^{27}$ But in this study urinary NGAL levels were not measured due to difficulties in the collection of rat's urine. These studies are without doubt corresponding with findings of this study.

Cystatin-C serum level was significantly increased in gentamicin group compared with the control group as supported by Kader et al.'s ${ }^{28}$ study that confirmed significant elevation in cystatin-C serum level during gentamicin induced-nephrotoxicity.

Cystatin-c is a surrogate biomarker of GFR, not affected by muscle mass, age, gender and food type, it superior to serum creatinin since; it detect earlier renal injury 2 days prior to elevation of blood urea and serum creatinine. Normally, cystatin- $\mathrm{C}$ is filtered by glomeruli and reabsorbed by proximal convoluted tubules thus; elevation of serum cystatin-C indicate a glomerular damage while elevation of urinary cystatin-C indicating renal tubular damage. ${ }^{29,30}$ Therefore, gentamicin in this study led to full prone nephrotoxicity and acute renal injury through reduction of GFR and elevation of glomerular and tubular damage biomarkers in the experimental rats.

Co-administration of pomegranate with gentamicin in this study leads to significant reduction of gentamicin induced-nephrotoxicity through reduction of blood urea significantly and significant amelioration of estimated GFR as observed in Alimordan et al.'s ${ }^{31}$ study that showed the nephro-protective effect of pomegranate in attenuation of gentamicin nephrotoxicity.
Also, body weight and BMI changes were insignificant improved compared with the control group as demonstrated by Primarizky et al.s ${ }^{32}$ study which pointed out that pomegranate is effective in preservation of normal body weight in rats during gentamicin induced-nephrotoxicity.

Regarding the effect of pomegranate on the oxidative stress, lipid peroxidation and anti-oxidant potential, pomegranate led to insignificant reduction of lipid peroxidation marker (MDA) and significant elevation of anti-oxidant marker (GSH). These findings are inconsistent with different studies that confirmed the anti-oxidant effect of pomegranate in attenuation of nephrotoxicity. ${ }^{33,34}$

Insignificant effect of pomegranate on lipid peroxidation in this study may be due to short duration of the experiment study, small sample size which may affect the statistical results or due to MDA serum level variability. Also, ElArabey $^{35}$ confirmed the insignificant effect of high dose of pomegranate in prevention of cisplatin inducednephrotoxicity. Certainly, there are different studies regarding the dose dependent and the anti-oxidant potential in attenuation of drug induced-nephrotoxicity. Low dose of pomegranate illustrated a protective effect in male rats, while high dose does not showed this defending effect. As well, gender differences in the protective effect of pomegranate was observed in Nematbakhsh et al.'s ${ }^{36,37}$ studies that showed male rats were more prone to the nephrotoxicity. These factors were excluded in this study, since we used male rats and fixed dose of pomegranate.

Furthermore, pomegranate significantly reduced inflammatory and renal tubular injury biomarkers (KIM-1 and NGAL) due to significant nephroprotective effect and attenuation of gentamicin induced-nephrotoxicity. Boroushaki et al.'s ${ }^{33}$ experimental study showed that pre-treatment with pomegranate lead to dose-dependent nephroprotective effect. Likewise, pomegranate led to reduction of cystatin-C but not to insignificant level. Reduction of cystatin- $C$ serum level indicates a protective effect on the glomeruli, since cystatin- $\mathrm{C}$ serum level is correlated with GFR. Therefore, pomegranate reduces renal tubular injury and improves glomerular function in different models of drug induced-nephrotoxicity. ${ }^{34}$

The nephroprotective effect of pomegranate is due to different constituents including gallic acid, punicallin, punicalgin, and ellagic acid which exhibit potent free radicals scavenging and anti-oxidant effects. ${ }^{38}$ Moreover, gallic acid exhibits significant nephroprotective effect in attenuation of vancomycin nephrotoxicity. ${ }^{39}$ But in this study active constituents of pomegranate juice were not determined as it is well evaluated in different studies. It has been shown that antioxidants ameliorate oxidative stress marker (MDA) but a higher dose of these anti-oxidants may induced oxidative stress causing augmentation of renal ischemic-reperfusion injury. ${ }^{40}$ This finding might explain insignificant effect of pomegranate on the MDA levels in this study, since we used a high dose of pomegranate $(100 \mathrm{mg} / \mathrm{kg})$.

Pomegranate therapy leads to significant weight reduction due to inhibition of digestive enzymes by tannis that lead to appetite suppression and reduction of nutrients absorption ${ }^{41}$ which might clarify the reduction in rat body weight in this study. 


\section{Conclusion}

Pomegranate attenuates gentamicin induced-nephrotoxicity through potentiation of endogenous anti-oxidant capacity and inhibition of inflammatory pathway.

\section{Acknowledgment}

The authors would like to thank the Research Deputy of International Campus, Al-Mustansiriyia University College of Medicine.

\section{Conflict of Interest}

None.

\section{Funding and Sponsorship}

None.

\section{References}

1. Tiong HY, Huang P, Xiong S, Li Y, Vathsala A, Zink D. Drug-induced nephrotoxicity: clinical impact and preclinical in vitro models. Mol Pharm. 2014:11:1933-1948.

2. Ennulat D, Ringenberg M, Frazier KS. Toxicologic pathology forum opinion paper: recommendations for a tiered approach to nonclinical mechanistic nephrotoxicity evaluation. Toxicol Pathol. 2018;46:636-646.

3. Brocca A, Virzì GM, Pasqualin C, Pastori S, Marcante S, de Cal M, et al. Cardiorenal syndrome type 5: in vitro cytotoxicity effects on renal tubular cells and inflammatory profile. Anal Cell Pathol (Amst). 2015:2015:469461.

4. Kim SY, Moon A. Drug-induced nephrotoxicity and its biomarkers. Biomol Ther (Seoul). 2012;20:268-272

5. A V, S A, Kuriakose J, Midhun SJ, Jyothis M, Latha MS. Protective effect of Rotula aquatica Lour against gentamicin induced oxidative stress and nephrotoxicity in Wistar rats. Biomed Pharmacother. 2018;106:1188-1194.

6. Moffett BS, Morris J, Galati M, Munoz FM, Arikan AA. Population pharmacokinetic analysis of gentamicin in pediatric extracorporeal membrane oxygenation. Ther Drug Monit. 2018;40:581-588.

7. Ge S, Beechinor RJ, Hornik CP, Standing JF, Zimmerman K, CohenWolkowiez M, et al. External evaluation of a gentamicin infant population pharmacokinetic model using data from a national electronic health record database. Antimicrob Agents Chemother. 2018;62. pii: 00669-18.

8. Aly HAA, Hassan MH. Potential testicular toxicity of gentamicin in adult rats Biochem Biophys Res Commun. 2018:497:362-367.

9. Moreira MA, Nascimento MA, Bozzo TA, Cintra A, da Silva SM, Dalboni MA, et al. Ascorbic acid reduces gentamicin-induced nephrotoxicity in rats through the control of reactive oxygen species. Clin Nutr. 2014;33:296-301.

10. Magee PJ, Rowland IR. Phyto-oestrogens, their mechanism of action: current evidence for a role in breast and prostate cancer. Br J Nutr. 2004;91:513-531.

11. Al-Kuraishy HM. Central additive effect of Ginkgo biloba and Rhodiola rosea on psychomotor vigilance task and short-term working memory accuracy. Journal of intercultural ethnopharmacology. 2016;5:7.

12. Huber R, Gminski R, Tang T, Weinert T, Schulz S, Linke-Cordes M, et al. Pomegranate (Punica granatum) seed oil for treating menopausal symptoms: an individually controlled cohort study. Altern Ther Health Med. 2017:23:28-34.

13. Akhtar S, Ismail T, Fraternale D, Sestili P. Pomegranate peel and peel extracts: chemistry and food features. Food Chem. 2015;174:417-425

14. Al-Kuraishy HM, Al-Gareeb Al. Potential effects of pomegranate on lipid peroxidation and pro-inflammatory changes in daunorubicin-induced cardiotoxicity in rats. Int J Prev Med. 2016:7:85.

15. Rasheed Z, Akhtar N, Anbazhagan AN, Ramamurthy S, Shukla M, Haqqi TM. Polyphenol-rich pomegranate fruit extract (POMx) suppresses PMAClinduced expression of pro-inflammatory cytokines by inhibiting the activation of MAP Kinases and NF-kappaB in human KU812 cells. J Inflamm (Lond). 2009:6:1.

16. Kim YE, Hwang CJ, Lee HP, Kim CS, Son DJ, Ham YW, et al. Inhibitory effect of punicalagin on lipopolysaccharide-induced neuroinflammation, oxidative stress and memory impairment via inhibition of nuclear factor-kappaB. Neuropharmacology. 2017:117:21-32.

17. Mansouri E, Basgen J, Saremy S. The effects of pomegranate extract on normal adult rat kidney: a stereological study. Vet Res Forum. 2016;7:1-6.

18. Singh AP, Junemann A, Muthuraman A, Jaggi AS, Singh N, Grover K, et al. Animal models of acute renal failure. Pharmacol Rep. 2012;64:31-44.

19. Gacka E, Życzkowski M, Bogacki R, Paradysz A, Hyla-Klekot L. The usefulness of determining neutrophil gelatinase-associated lipocalin concentration

excreted in the urine in the evaluation of cyclosporine A nephrotoxicity in children with nephrotic syndrome. Dis Markers. 2016;2016:6872149.

20. Abd-Elhamid TH, Elgamal DA, Ali SS, Ali FEM, Hassanein EHM, El-Shoura EAM, et al. Reno-protective effects of ursodeoxycholic acid against gentamicin-induced nephrotoxicitythrough modulation of NF-KB, eNOS and caspase-3 expressions. Cell Tissue Res. 2018;374:367-387.

21. Helal MG, Zaki MMAF, Said E. Nephroprotective effect of saxagliptin against gentamicin-induced nephrotoxicity, emphasis on anti-oxidant, antiinflammatory and anti-apoptic effects. Life Sci. 2018;208:64-71.

22. Ibraheem ZO, Basir R, Aljobory AKh, Ibrahim OE, Alsumaidaee A, Yam MF. Impact of gentamicin coadministration along with high fructose feeding on progression of renal failure and metabolic syndrome in Sprague-Dawley rats. Biomed Res Int. 2014;2014:823879.

23. Al-Kuraishy HM, Al-Gareeb Al, Al-Maiahy TJ. Concept and connotation of oxidative stress in preeclampsia. Journal of laboratory physicians. 2018;10:276

24. Hajihashemi S, Jafarian T, Ahmadi M, Rahbari A, Ghanbari F. Ameliorative effects of Zataria multiflora hydro-alcoholic extract on gentamicin induced nephrotoxicity in rats. Drug Res (Stuttg). 2018;68(7):387-394.

25. Luo QH, Chen ML, Sun FJ, Chen ZL, Li MY, Zeng W, et al. KIM-1 and NGAL as biomarkers of nephrotoxicity induced by gentamicin in rats. Mol Cell Biochem. 2014;397:53-60

26. Szeto CC, Kwan BC, Lai KB, Lai FM, Chow KM, Wang G, et al. Urinary expression of kidney injury markers in renal transplant recipients. Clin J Am Soc Nephrol. 2010;5:2329-2337.

27. Makris K, Markou N, Evodia E, Dimopoulou E, Drakopoulos I, Ntetsika K, et al. Urinary neutrophil gelatinase-associated lipocalin (NGAL) as an early marker of acute kidney injury in critically ill multiple trauma patients. Clin Chem Lab Med. 2009:47:79-82.

28. de Geus HR, Bakker J, Lesaffre EM, le Noble JL. Neutrophil gelatinaseassociated lipocalin at ICU admission predicts for acute kidney injury in adult patients. Am J Respir Crit Care Med. 2011:183:907-914.

29. Kader C, Sunbul M, Das YK, Yarim M, Bedir A, Karaca E, et al. Telbivudine attenuates gentamicin-induced kidney injury in rats. Int J Antimicrob Agents. 2017:49:595-602.

30. Al Suleimani YM, Abdelrahman AM, Karaca T, Manoj P, Ashique M, Nemmar A, et al. The effect of the dipeptidyl peptidase-4 inhibitor sitagliptin on gentamicin nephrotoxicity in mice. Biomed Pharmacother. 2018;97:11021108.

31. Alimoradian A, Changizi-Ashtiyani S, Ghiasabadi Farahani A, Kheder L, Rajabi R, Sharifi A. Protective effects of pomegranate juice on nephrotoxicity induced by captopril and gentamicin in rats. Iran J Kidney Dis. 2017;11:422429 .

32. Primarizky H, Yuniarti WM, Lukiswanto BS. Benefits of pomegranate (Punica granatum Linn) fruit extracts to weight changes, total protein, and uric acid in white rats (Rattus norvegicus) as an animal model of acute renal failure. Vet World. 2016;9:1269-1274

33. Boroushaki MT, Asadpour E, Sadeghnia HR, Dolati K. Effect of pomegranate seed oil against gentamicin-induced nephrotoxicity in rat. J Food Sci Technol. 2014:51:3510-3514

34. Cekmen M, Otunctemur A, Ozbek E, Cakir SS, Dursun M, Polat EC, et al. Pomegranate extract attenuates gentamicin-induced nephrotoxicity in rats by reducing oxidative stress. Ren Fail. 2013;35:268-274.

35. El-Arabey AA. Negative response of phytoestrogens of pomegranate flower extract against cisplatin-induced nephrotoxicity in female rats. Int J Prev Med. 2016;7:89 
36. Motamedi F, Nematbakhsh M, Monajemi R, Pezeshki Z, Talebi A, Zolfaghari $B$, et al. Effect of pomegranate flower extract on cisplatin-induced nephrotoxicity in rats. J Nephropathol. 2014;3:133-138.

37. Nematbakhsh M, Ebrahimian S, Tooyserkani M, Eshraghi-Jazi F, Talebi A, Ashrafi F. Gender difference in Cisplatin-induced nephrotoxicity in a rat model: greater intensity of damage in male than female. Nephrourol Mon. 2013;5:818-821.

38. Zhang L, Yang $X$, Zhang $Y$, Wang L, Zhang R. In vitro antioxidant properties of different parts of pomegranate flowers. Food Bioprod Process. 2011;89: 234-240.
39. Jang A, Srinivasan P, Lee NY, Song HP, Lee JW, Lee M, et al. Comparison of hypolipidemic activity of synthetic gallic acid-linoleic acid ester with mixture of gallic acid and linoleic acid, gallic acid, and linoleic acid on high-fat diet induced obesity in C57BL/6 Cr Slc mice. Chem Biol Interact. 2008;174:109-117.

40. Al-kuraishy HM, Al-Gareeb Al. Eustress and malondialdehyde (MDA): role of Panax ginseng: randomized placebo controlled study. Iranian journal of psychiatry. 2017;12:194.

41. Al-Gareeb Al, Aljubory KD, Alkuraishy HM. Niclosamide as an anti-obesity drug: an experimental study. Eating and Weight Disorders-Studies on Anorexia, Bulimia and Obesity. 2017;22:339-44.

This work is licensed under a Creative Commons Attribution-NonCommercial 3.0 Unported License which allows users to read, copy, distribute and make derivative works for non-commercial purposes from the material, as long as the author of the original work is cited properly. 